\title{
Proyección de los sistemas de cosecha forestal en las plantaciones establecidas en el contorno de la reserva Río Cruces para los próximos diez años
}

\author{
Projection of the forest harvesting systems in plantation areas established \\ in the contour of the Río Cruces reserve for the next ten years
}

\author{
Patricio Carey Ba*, Víctor Sandoval Va, Raúl Saldivia Ka \\ *Autor de correspondencia: ${ }^{a}$ Universidad Austral de Chile, Instituto de Manejo Forestal, Casilla 567, Valdivia, Chile, \\ pcarey@uach.cl
}

\begin{abstract}
SUMMARY
This study is a first report of the projection of the forest harvesting systems in plantation areas established at the contour of the Río Cruces reserve, located in the south of Chile. The study method considered the slope of the terrain at the plantation areas, the accessibility and the species. The study area was delimited and measured by a geographic information system; a set of thematic cover types was processed and obtained multiple graphical representations, which correspond to 9,472 ha. In the total area, a digital elevation model was processed in order to classify the harvesting areas in four slopes categories. This information allows to have a first approach in harvesting systems projection. Other data base was built related to areas and volumes of timber to be harvested for the plantations of Pinus radiata and Eucalyptus spp., during the next 10 years. The accessibility to these plantations includes principal paved roads and secondary gravel roads. The most relevant results were that $79 \%$ of the plantations in study area belongs to the forest companies. Besides, $90 \%$ of the total area $(8,553 \mathrm{ha})$ is in the slope range condition lower than $30 \%$. This indicated that the most important harvesting systems will be a ground-based mechanised system. The total harvesting volume projected in the plantation areas is approximately 2.4 million cubic meters in the next ten years.
\end{abstract}

Key words: forest harvesting, geographical information system, model of digital elevation.

\section{RESUMEN}

El presente trabajo es un avance de la proyección de los sistemas de cosecha forestal a utilizar en las plantaciones establecidas en el contorno de la reserva Río Cruces, considerando la pendiente del terreno, la accesibilidad y la especie. El área de estudio (9.472 ha) fue seleccionada y caracterizada mediante el apoyo de un sistema de información geográfico, con el cual se procesó un conjunto de coberturas temáticas con múltiples representaciones gráficas. Sobre esta superficie se construyó un modelo de elevación digital, se clasificó el terreno en cuatro categorías de pendiente y, de acuerdo con esta variable, se proyectaron los sistemas de cosecha forestal más adecuados. Además se cuantificaron las superficies y volúmenes a cosechar para las plantaciones de Pinus radiata y Eucalyptus spp., considerando un horizonte de proyección de 10 años. La accesibilidad a estas plantaciones comprende caminos principales pavimentados y caminos secundarios ripiados. Los resultados más relevantes fueron que el $79 \%$ de las plantaciones en estudio pertenecen a empresas forestales y que el $90 \%$ del área total (8.553 ha) posee pendientes inferiores al 30\%, condiciones que favorecen el trabajo de los sistemas de cosecha mecanizados con equipos de extracción terrestre como cortadoras, procesadoras y tractores skidders. El volumen total proyectado a cosechar es del orden de los 2,4 millones de metros cúbicos en el próximo período de 10 años.

Palabras clave: sistema de cosecha forestal, sistema de información geográfica, modelo de elevación digital.

\section{INTRODUCCIÓN}

Los sistemas de información geográfica (SIG) son una poderosa herramienta diseñada para el análisis de complejas interacciones espaciales (Ávila 1994). Esta herramienta tiene un amplio campo de aplicación en la cosecha fores- tal, actividad de gran impacto dentro del ciclo productivo forestal, dada su relación con los procesos industriales y su incidencia dentro de los costos de producción; es por ello que el adecuado manejo de la información, mediante esta herramienta, es relevante para la toma de decisiones acertadas en esta actividad (Arrué 1986). 
Una de las primeras etapas de la cosecha forestal se relaciona con la selección de la tecnología de producción a utilizar en un área de cosecha. Esto implica seleccionar los sistemas de cosecha más adecuados y eficientes para ser aplicados en relación a las características del terreno y las dimensiones del bosque. En la planificación de la cosecha se puede optar por sistemas de cosecha a base de equipos terrestres o de equipos aéreos, cuyas diferencias de operación están relacionadas directamente con las condiciones topográficas del sector, principalmente la pendiente. Por otra parte, es importante para la selección de la tecnología a utilizar, considerar las dimensiones de los árboles y la dimensión de los productos a obtener. También, para seleccionar el sistema de cosecha adecuado, se debe considerar la sensibilidad ambiental de las áreas de cosecha con el propósito de minimizar los impactos ambientales, tales como la estacionalidad de las operaciones.

Actualmente, en la zona sur de Chile, las empresas forestales utilizan principalmente tres tipos de sistemas de cosecha: altamente mecanizada, mecanizada tradicional y de cosecha aérea, los cuales representan los sistemas base de este estudio. La zona del contorno de la Reserva Nacional Río Cruces, ubicada en la provincia de Valdivia, en la zona sur de Chile, está dominada por praderas agrícolas y ganaderas con áreas de bosque nativo $\mathrm{y}$, actualmente, también existen plantaciones forestales, de las cuales no se posee una mayor referencia (CONAF-CONAMA 1999). Para esta zona, el objetivo del presente estudio fue proyectar los sistemas de cosecha a utilizar, durante los próximos 10 años, en la superficie de plantaciones forestales. Los resultados de este estudio pretenden orientar la toma de decisiones para la aplicación de los sistemas de cosecha forestal en la reserva Río Cruces, debido a la sensibilidad ambiental en esta área.

\section{MÉTODOS}

Área de estudio. La Reserva Nacional Río Cruces se ubica geográficamente entre los $39^{\circ} 34^{\prime}$ y $39^{\circ} 49^{\prime}$ latitud Sur y los $73^{\circ} 02^{\prime}$ y $73^{\circ} 18^{\prime}$ longitud Oeste. Con una superficie aproximada de 6.373 hectáreas, la reserva se ubica en la provincia de Valdivia y depende administrativamente de la Corporación Nacional Forestal (CONAF 1999) (figura 1). La composición del recurso forestal en la cuenca del río Cruces muestra una formación de renovales de especies nativas del tipo forestal siempreverde en los sectores más inmediatos a la reserva. Además existen áreas con plantaciones de especies exóticas, principalmente Pinus radiata D. Don y Eucalyptus spp., las cuales se han establecido junto a los rodales de especies nativas.

Información de soporte del estudio. En este estudio se utilizó el programa computacional Arc View versión 3.2 para representar datos georreferenciados, analizar las características y patrones de distribución de los datos y generar

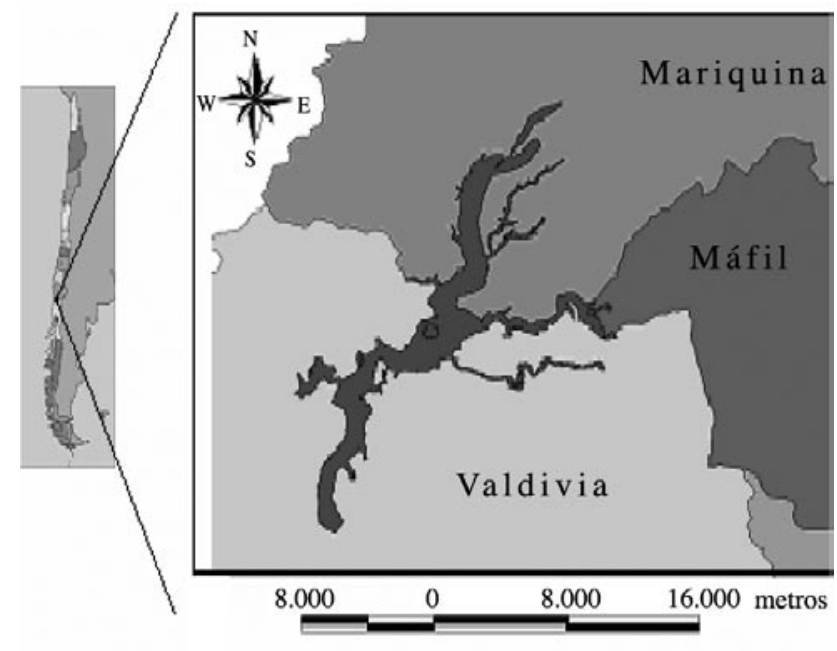

Leyenda

\section{Reserva Río Cruces}

Figura 1. Ubicación de la reserva Río Cruces.

Río Cruces reserve study area.

informes y mapas con los resultados de la información. Las coberturas de vegetación, de pendientes, de hidrografía y de caminos de la provincia de Valdivia fueron proporcionadas por el Laboratorio de Geomática del Instituto de Manejo Forestal de la Universidad Austral de Chile. Estas bases de información, escala 1:50.000, provienen del Catastro y Evaluación de Recursos Vegetacionales Nativos de Chile (CONAF-CONAMA 1999). Las coberturas de los límites de la reserva Río Cruces, escala 1:50.000, fueron proporcionadas por la Dirección Regional de la Corporación Nacional Forestal (CONAF) de Puerto Montt, mientras que las coberturas de los predios colindantes a la reserva fueron facilitadas por la Dirección Regional del Instituto de Desarrollo Agropecuario (INDAP). La información referida a las plantaciones forestales (especie y edad) fue facilitada por el Instituto Forestal (INFOR), específicamente del inventario regional realizado por esta entidad durante el año 2004. En el cálculo de los periodos de cosecha se consideró pertinente asumir para estas plantaciones los rangos de edades y sus respectivas proporciones de superficie según las estadísticas publicadas por INFOR (2004) para las plantaciones de $P$. radiata y Eucalyptus spp.

Delimitación de la zona estudio. Debido a que se trabajó con distintas fuentes de información, las cuales tenían diferentes datum, con lo que se producía un traslapo en las proyecciones de ciertas áreas, se procedió a homologar todos los datum al año 1984, con la finalidad de disminuir el error de proyección.

Para la determinación de los límites del área de estudio se trabajó, en primer lugar, con las coberturas de la reserva Río Cruces de curvas de nivel y de hidrología, específicamente seleccionando todos los cursos de agua que 
tributan hacia la reserva, con la finalidad de identificar la magnitud, su distribución y la influencia sobre la reserva forestal. De esta manera se determinó delimitar una faja de 3 mil metros de ancho en el contorno de los límites de la reserva Río Cruces, estableciendo de esta manera el límite del área de estudio.

A continuación se identificaron los diversos usos del suelo y, a través de la clasificación digital, se cuantificó la superficie de éstos en hectáreas. Posteriormente se procedió a identificar las áreas con plantaciones forestales ubicadas en esta zona base, cuantificando las superficies total y por especie. Se consideraron en este estudio únicamente las plantaciones de $P$. radiata, Eucalyptus globulus Labill. y Eucalyptus nitens Maiden. Para los predios pertenecientes a las empresas forestales se efectuó una verificación visual con una imagen satelital actualizada de la zona de estudio, con la finalidad de validar la existencia de la información de predios. Finalmente, se obtuvieron las superficies de los cursos de agua (principales y secundarios) y caminos (pavimentados y ripiados), con el propósito de cuantificar la proporción y magnitud de éstos.

Construcción del modelo de elevación digital (DEM). Se construyó el DEM con las pendientes de los sectores donde se encontraban establecidas las plantaciones forestales; esta cobertura de pendientes se elaboró de acuerdo con la información de curvas de nivel cada 25 metros de altura. Este modelo fue empleado para zonificar el área de trabajo en cuatro categorías de pendientes, con la finalidad de definir el sistema de cosecha más apropiado para cada condición. A continuación se describen las categorías de pendiente consideradas y los sistemas de cosecha apropiados por su funcionalidad y utilizados por las empresas forestales que prestan servicios de cosecha forestal en la zona sur de Chile. a) Sistema de cosecha terrestre en pendientes de 0\%-30\%: para pendientes de $0 \%$ a $15 \%$, son apropiados los sistemas altamente mecanizados (cortadora-procesadora, skidder grapple o forwarder); y en pendientes de $15 \%$ a $30 \%$ se utiliza principalmente el sistema mecanizado tradicional (skidder winche, motosierras). b) Sistemas de cosecha aérea o torres de madereo, utilizados en pendientes de $30 \%$ a $60 \%$. c) Terrenos con pendientes $>60 \%$ se consideran zonas de protección.

La resolución del DEM se controla con el tamaño de la grilla, la cual es definida como 100 m x 100 m (1 ha de superficie) para modelos representados con una escala de trabajo de 1:50.000.

Proyección de los sistemas de cosecha. El horizonte de proyección contempló un lapso de 10 años a partir de 2006 hasta el año 2015. Este rango fue divido en cuatro períodos, considerando como el primero de ellos el año 2006, posteriormente tres períodos consecutivos de tres años cada uno hasta el año 2015.

Teniendo en cuenta la clasificación de las pendientes en el área de estudio así como la superficie de plantacio- nes de $P$. radiata y Eucalyptus spp., se consideraron las edades de las plantaciones en cada predio con la finalidad de proyectar la cosecha de éstas. Para $P$. radiata el rango de edad de rotación fue de 22 a 25 años y para Eucalyptus spp. de 8 a 12 años, de acuerdo con las diversas edades de las plantaciones en los 172 predios analizados.

Una vez cuantificadas las superficies a cosechar para cada período se determinaron los volúmenes a cosechar por especie. Para esto se consideró un rendimiento promedio a la edad de cosecha forestal en la zona de Valdivia de $350 \mathrm{~m}^{3} /$ ha para $P$. radiata (INFOR 2004) y de $281 \mathrm{~m}^{3} / \mathrm{ha}$ para Eucalyptus spp. (Sotomayor et al. 2002).

\section{RESULTADOS}

Uso del suelo. El área de estudio, delimitada alrededor de la reserva Río Cruces, correspondió a 37.378 ha, en las cuales existen diversos usos del suelo. Sin embargo, la mayor proporción de superficie está ocupada por bosques con 17.588 ha, lo que equivale al $47,1 \%$ de la superficie total de la franja demarcada. Dentro de la categoría bosques están contemplados tanto las plantaciones como el bosque nativo. La accesibilidad a la zona de estudio, a través de caminos principales pavimentados y secundarios ripiados, se caracterizó por una densidad total de caminos de $27 \mathrm{~m} / \mathrm{ha}$. Los caminos principales tienen una mayor densidad (15 m/ha) en comparación con los caminos secundarios (12 m/ha). Los ríos solamente ocuparon un $2 \%$ de la superficie total, sin embargo, la mayor parte de los cursos de agua drenan hacia la Reserva Río Cruces.

En el área de estudio los bosques están constituidos por dos clases de recursos forestales, las plantaciones con 9.541 ha (figura 2), que equivalen al 54,2\% de esta categoría, y el bosque nativo, constituido por renovales y bosque adulto con 8.047 ha.

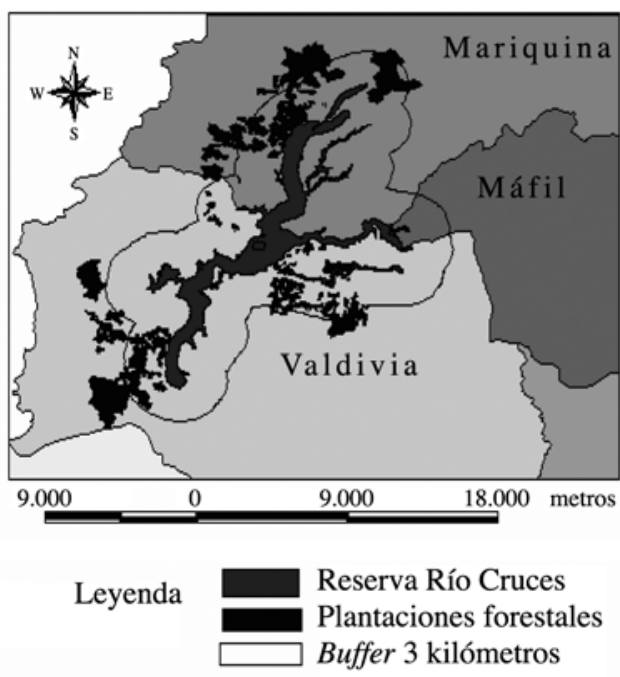

Figura 2. Localización de las plantaciones forestales en la zona de estudio.

Forest plantations at the study area. 
La especie que ocupó una mayor superficie dentro de la zona en estudio fue $P$. radiata con 6.425 ha, correspondiendo a un $67,3 \%$ del total, mientras que prácticamente toda la superficie restante está ocupada por Eucalyptus spp. con 3.047 ha. Dentro del área total de las plantaciones forestales las zonas de protección alrededor de los cursos de agua alcanzaron 8.799 ha (cuadro 1).

Cuadro 1. Zonas de protección asociadas con plantaciones forestales.

Riparian zones associated with forest plantations.

\begin{tabular}{lcc}
\hline Ríos & Longitud (km) & $\begin{array}{c}\text { Superficie } \\
\text { de protección (ha) }\end{array}$ \\
\hline Ríos principales & 48,7 & 292 \\
Ríos secundarios & 128,9 & 5.878 \\
Total & 177,6 & 8.799 \\
\hline
\end{tabular}

Fuente: CONAF-CONAMA (1999).

Distribución administrativa y tenencia de la tierra. La mayor superficie de las plantaciones forestales en el área de estudio se ubican en la comuna de Valdivia (6.651 ha), representando un 70,2\% del total del área. En la comuna de San José de la Mariquina se ubican 2.821 ha, que corresponden a un $29,8 \%$ de la superficie total. Por otra parte, en relación a la propiedad de los bosques, 7.436 ha correspondieron a las empresas forestales, mientras los pequeños propietarios poseían 1.741 ha en la zona de estudio (cuadro 2).

Cuadro 2. Superficie de plantaciones forestales según propietario y especie.

Forest plantations area by ownership and species.

\begin{tabular}{lrrrrr}
\hline & \multicolumn{2}{c}{ Pinus radiata } & & \multicolumn{2}{c}{ Eucalyptus spp. } \\
\cline { 2 - 3 } \cline { 6 - 7 } Propietarios & \multicolumn{1}{c}{ (ha) } & \multicolumn{1}{c}{$(\%)$} & & (ha) & \multicolumn{1}{c}{$(\%)$} \\
\hline Empresas forestales & 5.812 & 90,5 & & 1.624 & 53,3 \\
Pequeños propietarios & 613 & 9,5 & & 1.128 & 37,0 \\
Otros propietarios & 0,0 & 0,0 & & 295 & 9,7 \\
Total & 6.425 & 100,0 & & 3.047 & 100,0 \\
\hline
\end{tabular}

Fuente: INFOR (2004).

Zonificación de acuerdo a la pendiente. La mayor proporción de la superficie (90\%) correspondió a terrenos bajo 30\% de pendiente, lo que indica que los equipos de cosecha mediante extracción terrestre serán los de mayor aplicación para las futuras cosechas forestales en el área de estudio. Específicamente 5.320 ha del área total poseían pendientes entre 0 y $15 \%$, las cuales, de acuerdo a la funcionalidad de la tecnología actual deberían ser cosechadas con equipos altamente mecanizados, tales como cortadoras hidráulicas, skidder grapple, forwarders y camiones tronqueros. La superficie con pendientes entre $15 \%$ y $30 \%$ correspondió a 3.233 ha, las que deberían ser trabajadas con sistemas de cosecha mecanizada convencional, tales como motosierras en volteo, skidder con winche y camión con carro. Cabe señalar que la superficie ocupada por $P$. radiata y Eucalyptus spp. disminuyó drásticamente al aumentar el porcentaje de pendiente del terreno (cuadro 3).

Cuadro 3. Superficie de plantaciones (ha) por clases de pendiente y especie.

Forest plantations area (ha) by slope class and species.

\begin{tabular}{lccr}
\hline Pendiente $(\%)$ & P. radiata & Eucalyptus spp. & Total \\
\hline $0-15$ & 3.937 & 1.383 & 5.320 \\
$15-30$ & 2.000 & 1.233 & 3.233 \\
$30-60$ & 440 & 406 & 846 \\
$>60$ & 48 & 25 & 73 \\
Total & 6.425 & 3.047 & 9.472 \\
\hline
\end{tabular}

Proyección de volúmenes de cosecha. El volumen total en el área de estudio, proyectado a la cosecha en la edad de rotación de las especies, fue de $3.081 .132 \mathrm{~m}^{3}$, cuya cifra está compuesta en un mayor porcentaje $(72,4 \%)$ por los aportes volumétricos de las trozas de $P$. radiata. Del total proyectado a cosechar, $1.766 .573 \mathrm{~m}^{3}$ correspondieron a la clase inferior de pendiente $(0 \%-15 \%)$ equivalente a un $57,3 \%$ del volumen total (cuadro 4 ).

Cuadro 4. Volumen total $\left(\mathrm{m}^{3}\right)$ proyectado a cosechar por clase de pendiente y especie.

Total harvesting volume $\left(\mathrm{m}^{3}\right)$ projected by slope class and species.

\begin{tabular}{lrcr}
\hline Pendiente $(\%)$ & P. radiata & Eucalyptus spp. & \multicolumn{1}{c}{ Total } \\
\hline $0-15$ & 1.377 .950 & 388.623 & 1.766 .573 \\
$15-30$ & 700.000 & 346.473 & 1.046 .473 \\
$30-60$ & 154.000 & 114.086 & 268.086 \\
Total & 2.231 .950 & 849.182 & 3.081 .132 \\
\hline
\end{tabular}

Considerando el período de proyección de 10 años, al año 2015 se obtiene la cosecha de la mayor superficie de plantaciones forestales, alcanzando a 2.221 ha de $P$. radiata. Por otra parte, en el período 2010-2012 se obtiene la menor superficie a cosechar de $P$. radiata, con sólo 52 ha (cuadro 5).

Cuadro 5. Superficie (ha) y volumen total $\left(\mathrm{m}^{3}\right)$ proyectados a cosechar por especie, según período.

Total projected harvesting area (ha) and volume $\left(\mathrm{m}^{3}\right)$ by species, according to period.

\begin{tabular}{lrrrrr}
\hline & \multicolumn{2}{c}{ Pinus radiata } & & \multicolumn{2}{c}{ Eucalyptus spp. } \\
\cline { 2 - 3 } \cline { 5 - 6 } Período (años) & \multicolumn{1}{c}{ (ha) } & \multicolumn{1}{c}{$\left(\mathrm{m}^{3}\right)$} & & (ha) & \multicolumn{1}{c}{$\left(\mathrm{m}^{3}\right)$} \\
\hline $1(2006)$ & 501 & 175.350 & & 631 & 177.311 \\
$2(2007-2009)$ & 1.490 & 521.500 & & 664 & 186.584 \\
$3(2010-2012)$ & 52 & 18.200 & & 1.399 & 393.119 \\
$4(2013-2015)$ & 2.221 & 777.350 & & 631 & 177.311 \\
Total & 4.264 & 1.492 .400 & & 3.325 & 934.325 \\
\hline
\end{tabular}


Para Eucalyptus spp., en el tercer período (2010-2012) se proyectan las mayores superficies a cosechar (1.399 ha) y en los otros períodos la superficie a cosechar alcanza niveles algo superiores a 600 ha (cuadro 5).

\section{DISCUSIÓN Y CONCLUSIONES}

Con respecto a la superficie de plantaciones forestales por especie se puede apreciar que la mayor superficie la ocupa $P$. radiata $(67,3 \%)$ en relación a Eucalyptus spp. Sin embargo, al comparar estos valores con la superficie de plantaciones existentes hace 10 años en la provincia de Valdivia, se observa un cambio en esta proporción, debido al aumento de la superficie plantada con Eucalyptus spp. en terrenos que anteriormente eran ocupados por $P$. radiata (INFOR 1996).

En relación a la tenencia de la tierra en el área de estudio, las empresas forestales son las que poseen la mayor superficie de las plantaciones forestales $(78,5 \%)$. Por tal razón, la proyección de los sistemas de cosecha a utilizar está enfocada principalmente en los terrenos que pertenecen a esta clase de propietario.

El $90 \%$ de la superficie total de las plantaciones forestales está ubicado en terrenos con pendientes inferiores al $30 \%$. Esto equivale a 8.553 ha, lo que indica que, desde el punto de vista de cosecha y transporte, estas áreas presentan ventajas, ya que representan un menor costo de producción por metro cúbico al utilizar sistemas de cosecha mediante extracción terrestre. Cabe resaltar que los sistemas antes mencionados presentan una mayor productividad (volumen/hora), no obstante que presentan limitaciones en la estacionalidad de las operaciones, debiendo utilizarse sólo en temporada seca o estival.
El volumen total proyectado a cosechar, para ambas especies a la edad de rotación es de $3.081 .132 \mathrm{~m}^{3}$, considerando la productividad de toda la superficie de plantaciones forestales, con pendientes inferiores al 60\% (9.389 ha). No obstante, el volumen total a cosechar durante el periodo de proyección es de $2.426 .725 \mathrm{~m}^{3}$, ya que no toda la superficie de $P$. radiata cumplirá con la edad de rotación para ser cosechada en los próximos 10 años.

\section{REFERENCIAS}

Arrué E. 1986. Funciones de tiempo y rendimiento en faenas de explotación forestal. Concepción, Chile. CORMA (Chile). $193 \mathrm{p}$.

Ávila A. 1994. Comparación de modelos de elevación digital de un sistema de información geográfico, a partir de la cartografía escala 1:25.000 y 1:50.000. Tesis Ingeniero Forestal. Valdivia, Chile. Facultad de Ciencias Forestales, Universidad Austral de Chile. 45 p.

CONAF-CONAMA-BIRF (Corporación Nacional Forestal Corporación Nacional del Medio Ambiente, CL). 1999. Catastro y Evaluación de recursos vegetacionales nativos de Chile. 137 p. (Informe Regional Décima Región de Los Lagos).

CONAF (Corporación Nacional Forestal, CL). 1999. Plan de manejo Reserva Nacional Río Cruces. Ministerio de Agricultura, Corporación Nacional Forestal, Programa Patrimonio Silvestre. Chile. 214 p. (Documento de Trabajo $N^{\circ} 325$ ).

INFOR (Instituto Forestal, CL). 1996. Estadísticas forestales 1996. Región de Los Lagos. Santiago, Chile. 151 p. (Boletín Estadístico 48).

INFOR (Instituto Forestal, CL). 2004. Estadísticas forestales 2004. Región de Los Lagos. Santiago, Chile. 127 p. (Boletín estadístico 103).

Sotomayor A, E Helmke, E García. 2002. Manejo y mantención de plantaciones forestales. Concepción, Chile. INFOR, CORFO (Chile). 51 p. 